\title{
HOMOGENEOUS SPACES WITH VANISHING STEENROD SQUARING OPERATIONS
}

\author{
VICTOR SCHNEIDER
}

ABSTRACT. If $G$ is a compact, connected Lie group, $H$ is a closed subgroup of $G$ and $G / H$ has no nonzero Steenrod operations, then $G / H$ splits as a product of homogeneous spaces of simple Lie groups (the factors of $G$ ). This fact is used to classify transitive actions on spaces with vanishing Steenrod operations, namely product of certain Stiefel manifolds and spheres.

1. Introduction. A homogeneous space $M$ is a differentiable manifold which admits a transitive, differentiable action by a compact, connected Lie group $G$. Any such $G$ may be uniquely expressed as $G=\bar{G} / N=\bar{G}_{1} \times \cdots \times$ $\bar{G}_{s} / N$ where $\bar{G}_{i}$ is either $S^{1}$ or a simple, simply connected Lie group and $N$ is a finite normal subgroup of $\bar{G}$. If $H$ is an isotropy subgroup of the action of $G$ on $M$ and $\operatorname{Rk}(H)=\operatorname{Rk}(G)$, it is well known that $G=G_{1} \times \cdots \times G_{s}$ $\left(G_{i}=\bar{G}_{i} / N_{i}, N_{i}=\bar{G}_{i} \cap N\right)$ and $M$ is diffeomorphic to $G_{1} / H_{1} \times \cdots \times G_{s} / H_{s}$ where $H_{i}=G_{i} \cap H$. If $\operatorname{Rk}(G)>\operatorname{Rk}(H)$, the above splitting may not occur. With the assumption that the action of $G$ is irreducible (no proper normal subgroup of $G$ acts transitively on $M$ ) the following results have been established: $M$ does split as a product if $(1) H^{*}(M ; Q)$ is a Hopf algebra with some dimensional restrictions [3], or (2) $M$ is highly connected relative to $\mathrm{Rk}(G)-\mathrm{Rk}(H)[7]$. In this paper we prove:

Theorem 1. Let $M$ be 11-connected and have all trivial mod-2 Steenrod squaring operations. If there is an irreducibly transitive, effective action of $G$ on $M, M$ is diffeomorphic to $G_{1} / H_{1} \times \cdots \times G_{s} / H_{s}$ where the $G_{i}$ are simple factors of $G$ and $H_{i}=G_{i} \cap H$.

As in [3], [7] this splitting (or decomposition) theorem can be used to classify some homogeneous spaces.

Received by the editors April 30, 1973 and, in revised form, May 2, 1974. AMS (MOS) subject classifications (1970). Primary 57E47.

Key words and phrases. Homogeneous space, Steenrod operations, spectral sequence. 
Theorem 2. If $M$ is an 11-connected homogeneous space with vanishing Steenrod squaring operations, then $M$ can only be a product of spheres and the Stiefel manifolds $V_{n, n-2}=\mathrm{SO}(n) / \mathrm{SO}(n-2), w_{n, n-2}=\mathrm{SU}(n) / \mathrm{SU}(n-2)$ and $X_{n, n-2}=\operatorname{Sp}(n) / \mathrm{Sp}(n-2)$ with $n$ even.

Theorem 3. If $M$ is a homogeneous space which has the same homotopy type as a product of spheres, all of dimension greater than 11 , then $M$ is diffeomorphic to that product of standard spheres and any effective, irreduc. ibly transitive action on $M$ is a product of the known actions on each sphere.

The condition that $M$ be 11 -connected in Theorem 1 is necessary as the following example shows: Let $G=\mathrm{Sp}(3) \times \mathrm{Sp}(n)$ with $n$ even and $n>4$. Now there are standard embeddings $\phi_{1}: \mathrm{Sp}(2) \rightarrow \mathrm{Sp}(3)$ and $\phi_{2}: \mathrm{Sp}(2) \times \mathrm{Sp}(n-2)$ $\rightarrow \mathrm{Sp}(n)$ so we can define $\phi: \mathrm{Sp}(2) \times \mathrm{Sp}(n-2) \rightarrow \mathrm{Sp}(2) \times \mathrm{Sp}(n)$ by $\phi\left(g_{1}, g_{2}\right)$ $=\left(\phi_{1}\left(g_{1}\right), \phi_{2}\left(g_{1}, g_{2}\right)\right)$. If $H$ is the image of $\phi, G / H$ is 10 -connected, has all trivial Steenrod operations, and the action is not a product action. Theorem 3 extends Theorem $B$ of [7] to a much larger class of products of spheres.

2. Preliminaries. We will consider only $Z_{2}$-cohomology and $G$ will always act irreducibly. If $G$ acts transitively and effectively on $M$, then $\bar{G}$ has a natural transitive, almost effective action on $M$. Just as the action of $G$ on $M$ can be represented as translation on $G / H$, the action of $\bar{G}$ on $M$ can be represented as translation on $\bar{G} / \bar{H}$.

The mod 2-cohomology of the simply connected, simple classical groups is as follows [2], [6]:

$H^{*}(\operatorname{Spin}(n))$ has a simple system of generators $w_{3}, \ldots, w_{n-1}, u$, where $\operatorname{dim} w_{i}=i, w_{i} \cup w_{i}=w_{2 i}$ if $2 i \leq n-1$,

$\operatorname{dim} u=t-1$ ( $t$ is the smallest power of 2 larger than $n-1)$, $w_{i}=0$ if $i$ is a power of 2 , and

$\mathrm{Sq}^{i}\left(w_{j}\right)=C_{i}^{j} w_{j+i}$, if $i+j \leq n-1$, where $C_{i}^{j}$ is the binomial coefficient reduced $\bmod 2$.

$H^{*}(\mathrm{SU}(n))$ and $H^{*}(\mathrm{Sp}(n))$ are exterior algebras with generators $w_{d j+d-1}$, $j=1, \ldots, n-1$ for $\mathrm{SU}(n)$ and $j=0, \ldots, n-1$ for $\operatorname{Sp}(n)$, and $\operatorname{dim} w_{d j+d-1}=d j+d-1$, where $d=2$ for $\operatorname{SU}(n)$ and $d=4$ for $\operatorname{Sp}(n)$,

$$
\begin{aligned}
\mathrm{Sq}^{d i} w_{d j+d-1} & =C_{i}^{j} w_{d(j+i)+d-1} & & \text { for } j+i \leq n-1, \\
& =0 & & \text { otherwise. }
\end{aligned}
$$

We organize some facts in the following: 
Lemma 2.1. Let $\bar{G} / \bar{H}$ be 11-connected with $\bar{G}$ acting irreducibly. Then all the factors of $\bar{G}$ have rank 4 or greater and all the factors of $\bar{H}$ have rank 3 or greater. For each Spin, SU or Sp factor of $\bar{G}$, there is a corresponding Spin, SU or Sp factor of $\bar{H}$ which projects nontrivially onto it. Also the only possible exceptional factor of $\bar{G}$ is $E_{8}$ and then $\bar{H}$ must have a factor $E_{7}$ which projects into it.

Proof. By Lemmas 2.1 and 2.2 of [7], $i: \bar{H} \rightarrow \bar{G}$ induces an isomorphism through $\operatorname{dim} 10$, in cohomology, and $\bar{G}$ and $\bar{H}$ have the same number of simple factors. Let $\bar{G}_{i}$ be a factor of $\bar{G}$ and $p_{i}: \bar{G} \rightarrow \bar{G}_{i}$ the projection. It was pointed out in [7] that the action of $\bar{G}$ is not irreducible if $p_{i}(\bar{G})=\bar{G}_{i}$, and in the proof of $\left[7\right.$, Theorem A] it was shown that $p_{i}(\bar{H})=\bar{G}_{i}$ if $\bar{G} / \bar{H}$ is $11-$ connected and $\bar{G}_{i}$ has rank less than four. The $Z_{2}$-structure of the exceptional groups can be found in [8]. Since $F_{4}$ is the only simple group with an indivisible generator in $\operatorname{dim} 4, F_{4}$ is a factor of $\bar{G}$ if and only if $F_{4}$ is a factor of $\bar{H}$. But then the projection of $\bar{G}$ onto an $F_{4}$ factor would carry $\bar{H}$ onto that factor, which again is impossible since $\bar{G}$ acts irreducibly. Since $E_{6}, E_{7}$ and $E_{8}$ all have an indivisible generator in dim 9 which is not the Steenrod square of a generator in $\operatorname{dim} 7$ and the classical groups do not, $\bar{G}$ has an $E$ factor if and only if $\bar{H}$ does. Since $E_{6}$ has no generator in $\operatorname{dim} 10$ and $E_{7}$ and $E_{8}$ do, the only possibility is that $\bar{G}$ may have an $E_{8}$ factor and then $\bar{H}$ must have an $E_{7}$ factor. For each Sp factor of $\bar{G}$ we have a generator $w_{3}$ of $H^{3}(\bar{G})$ such that $\mathrm{Sq}^{2} w_{3}=0$ and $w_{3} \cup w_{3}=0$. Hence the isomorphism $i^{*}$ must carry $w_{3}$ onto a generator (possibly modulo some other elements) with the same properties. Hence $\bar{H}$ must have an Sp factor which projects nontrivially into the Sp factor of $\bar{G}$. For the SU case, we have a generator $w_{3}$ such that $\mathrm{Sq}^{2} w_{3} \neq 0$ and $w_{3} \cup w_{3}=0$ and for the Spin case we have a $w_{3}$ with $\mathrm{Sq}^{2} w_{3} \neq 0$ and $w_{3} \cup w_{3} \neq 0$, so these cases are handled similarly. Since $\bar{G} / \bar{H}$ is 11 -connected, $i^{*}$ is also injective in $\operatorname{dim}$ 11. Noting the restrictions on the factors of $\bar{G}$ and the cohomology of the classical groups listed above, we see that the factors of $\bar{H}$ must have rank at least three.

3. Proofs. Suppose $M$ (or $\bar{G} / \bar{H}$ ) does not split as a product. Let $q$ be the first dimension past zero where $H^{q}(M) \neq 0$. By Lemma 2.1 of [7], $i: \bar{H} \rightarrow$ $\bar{G}$ induces an isomorphism through $\operatorname{dim} q-2$ and $i^{*}$ is injective in $\operatorname{dim}$ $q-1$. If $q$ is a power of two and $\operatorname{Spin}(q)$ is a factor of $\bar{H}$, we have the "extra" generator $u \in H^{q-1}($ Spin $(q))$ and so it is possible that $\operatorname{dim} H^{q-1}(\bar{G})$ $<\operatorname{dim} H^{q-1}(\bar{H})$. If, however, $q$ is not a power of two, there are no "extra" 
generators in $\operatorname{dim} q-1$; and, since $\bar{G}$ and $\bar{H}$ have the same number of factors, $\operatorname{dim} H^{q-1}(\bar{G})=\operatorname{dim} H^{q-1}(\bar{H})$.

We first consider the case when $q$ is not a power of two; i.e., when $i^{*}$ is an isomorphism through $\operatorname{dim} q-1$. Hence $p^{*}: H^{q}(M) \rightarrow H^{q}(\bar{G})$ is injective where $p: \bar{G} \rightarrow M$ is the projection of the bundle $\bar{H} \rightarrow \bar{G} \rightarrow M$. If $0 \neq$ $v \in H^{q}(M), p^{*}(v)$ cannot be a divisible element in $H^{q}(\bar{G})$ because this would imply that $i^{*} p^{*}(v) \neq 0$. So, if $j: \bar{G}_{j} \rightarrow \bar{G}$ is inclusion as a factor, $j^{*} p^{*}(v)$ must be an indivisible element (possibly modulo some divisible elements) in the cohomology of one of the factors of $\bar{G}$.

Let us first consider the case when $j^{*} p^{*}(v)$ is nonzero in a Spin factor. If $q$ is odd and $q+1$ is not a power of two, $\mathrm{Sq}^{1} w_{q}=w_{q+1}$ in $H^{*}$ (Spin). But then by naturality of the Steenrod squares, $\mathrm{Sq}^{1}(\nu)$ is nonzero unless $w_{q+1}=0$. This means that the Spin factor in question can only be $\operatorname{Spin}(q+1)$. If $q$ is odd and $q+1$ is a power of two, $\mathrm{Sq}^{2} w_{q}=w_{q+2}$. We also have $\mathrm{Sq}^{2} w_{q}=w_{q+2}$ if $q$ is even, not divisible by four and $q+2$ is not a power of two. Hence $w_{q+2}=0$ (for otherwise $\mathrm{Sq}^{2} v \neq 0$ by naturality) and the Spin factor can only be $\operatorname{Spin}(q+1)$ or $\operatorname{Spin}(q+2)$. If $q$ is even, not divisible by four and $q+2$ is a power of two, $\mathrm{Sq}^{4} w_{q}=w_{q+4}$ and we get $\operatorname{Spin}(q+3)$ and $\operatorname{Spin}(q+4)$ as possible factors. The case where $q$ is divisible by four is technically more difficult. Let $\operatorname{Spin}(n)$ be the factor and consider $w_{q-1}, w_{q}, w_{q+1} \epsilon$ $H^{*}(\operatorname{Spin}(n))$. Suppose $i^{*}\left(w_{q+1}\right) \neq 0$ in $H^{*}(\bar{H})$. (We identify the $w^{\prime}$ s with elements in $H^{*}(\bar{G})$.) Since $\mathrm{Sq}^{2} w_{q-1}=w_{q+1}, \operatorname{Sq}^{2}\left(i^{*}\left(w_{q-1}\right)\right)=i^{*} w_{q+1}$. Hence $i^{*}\left(w_{q-1}\right)$ and $i^{*}\left(w_{q+1}\right)$ must project into the same factor of $\bar{H}$. Since $\mathrm{Sq}^{1} w_{q-1}^{q-1}=w_{q}$ and $i^{*} w_{q}=0, \mathrm{Sq}^{1}\left(i^{*}\left(w_{q-1}\right)\right)=0$; this means that the factor of $\bar{H}$ is not a Spin factor. Since the generators $i^{*}\left(w_{q-1}\right)$ and $i^{*}\left(w_{q+1}\right)$ are two dimensions apart, the factor cannot be an $\mathrm{Sp}$. So it must be an SU factor and must in fact be at least $\mathrm{SU}((q+2) / 2)$. By Lemma $2.1, \bar{H}$ has a Spin factor which projects into $\operatorname{Spin}(n)$. Since $i^{*}$ is an isomorphism through dim $q-1$, this Spin factor must be at least a $\operatorname{Spin}(q)$. So the image of $\bar{H}$ under the projection into Spin $(n)$ contains a subgroup locally isomorphic to $\operatorname{Spin}(q) \times \operatorname{SU}((q+2) / 2)$. Now let $q=k t$ where $k$ is odd and $t$ is a power of two. Since $C_{t}^{q}=C_{q-t}^{q} \neq 0 \bmod 2$, we have $\mathrm{Sq}^{t} w_{q}=w_{q+t}$ if $q+t$ is not a power of two and $\mathrm{Sq}^{q-t} w_{q}=w_{2 q-t}$ if $q+t$ is a power of two. So as before $w_{q+t}$ or $w_{2 q-t}$ must be zero respectively. This gives the restriction $n \leq$ $\max \{q+t, 2 q-t\} \leq 2 q-4$. But

$$
\mathrm{Rk}\left(\operatorname{Spin}(q) \times \mathrm{SU}\left(\frac{q+2}{2}\right)\right)=\frac{q}{2}+\frac{q}{2}>q-2 \geq \mathrm{Rk}(\operatorname{Spin}(n)) .
$$


Faced with this contradiction, we conclude that $i^{*}\left(w_{q+1}\right)=0$. But now if we examine the spectral sequence for $\bar{H} \rightarrow \bar{G} \rightarrow M$, we see that there must be a nonzero element $z \in H^{q+1}(M)$ such that $j^{*} p^{*}(z)=w_{q+1}$, modulo some divisible elements. But $\mathrm{Sq}^{1}\left(w_{q+1}\right)=w_{q+2}$, hence $w_{q+2}$ must be zero and $n=q+1$ or $q+2$. In summary, we have established that if $j^{*} p^{*}(v)$ is nonzero in a Spin factor with $q$ not a power of two, the factor is $\operatorname{Spin}(q+i)$, $i=1,2,3,4$. Also, since $i^{*}$ is an isomorphism through $\operatorname{dim} q-1$, any Spin factor of $\bar{H}$ must be at least a $\operatorname{Spin}(q)$. By Lemma 2.1, a $\operatorname{Spin}(q)$ factor of $\bar{H}$ must project nontrivially into $\operatorname{Spin}(q+i)$; and, since all factors of $\bar{H}$ have rank three or greater, the image of $\bar{H}$ has no other factor.

The cases where $j^{*} p^{*}(v)$ is nonzero in an SU or Sp factor are similar. Write $q=d k+d-1$ where $d=2$ or 4 if $j^{*} p^{*}(v)$ is nonzero in an SU or Sp factor, respectively. The argument given above is repeated using $\mathrm{Sq}^{d} w_{q}$ if $k$ is odd, $\mathrm{Sq}^{d 2} w_{q}$ if $k$ is even but not divisible by four and $\mathrm{Sq}^{d} w_{q+d}$ if $k$ is divisible by four. No special care has to be taken if $k, k+1$ or $k+2$ is a power of two. Hence we get only $\mathrm{SU}((q+1) / 2), \operatorname{SU}((q+3) / 2)$, $\mathrm{Sp}((q+1) / 4)$ or $\mathrm{Sp}((q+5) / 4)$ as possible factors of $\bar{G}$ and $\mathrm{SU}((q-1) / 2)$ or $\mathrm{Sp}((q-3) / 4)$ as possible factors of $\bar{H}$ which project nontrivially into the $\mathrm{SU}$ or Sp factors, respectively.

By Lemma $2.1, E_{8}$ is the only possible factor of $\bar{G}$ and $E_{7}$ must project into it. Using the information listed in [8], we see that an isomorphism from $H^{*}\left(E_{8}\right)$ to $H^{*}\left(E_{7}\right)$ through $\operatorname{dim} 10$ must extend to an isomorphism through $\operatorname{dim} 14$. But $\operatorname{dim} H^{15}\left(E_{8}\right)=\operatorname{dim} H^{15}\left(E_{7}\right)+1$. If $q=15$ and $j^{*} p^{*}(v)$ is nonzero in $H^{15}\left(E_{8}\right)$, it can only map onto elements in $H^{15}\left(E_{8}\right)$ which have nonzero Steenrod squares. Thus the exceptional groups may be eliminated.

Now we must dispose of the case when $q$ is a power of two. Since $H^{q}(\bar{G})$ has only divisible elements, $p^{*}(v)=0$ for $v \in H^{q}(M)$. Also $i^{*}$ is an isomorphism through $\operatorname{dim} q-2$ and injective in $\operatorname{dim} q-1$. Studying the spectral sequence, we see that this is only possible if $\operatorname{dim} H^{q-1}(\bar{G})<$ $\operatorname{dim} H^{q-1}(\bar{H})$ and the differential $d_{0, q-1}^{q}: H^{q-1}(\bar{H}) \rightarrow H^{q}(M)$ is onto. This implies that one of the factors of $\bar{H}$ must be $\operatorname{Spin}(q)$ as checking the cohomology listed above will show. But now with the same argument we used when $q$ was divisible by four, we see that there is a nonzero element $z \epsilon$ $H^{q+1}(M)$ such that $j^{*} p^{*}(z)=w_{q+1}$, modulo some divisible elements, in $H^{*}$ (Spin). But $\mathrm{Sq}^{1} w_{q+1}=w_{q+2}$, so the Spin factor must again be $\operatorname{Spin}(q+1)$ or $\operatorname{Spin}(q+2)$.

Rewrite $\bar{G}=G_{1} \times \bar{G}_{1}$ where $G_{1}$ is the factor of $\bar{G}$ selected above, and 
let $H_{1}$ be the factor of $\bar{H}$ which projects into $G_{1}$. Let $\Gamma_{1}$ be the image of the homomorphism obtained by composing the inclusion $H_{1} \rightarrow \bar{H}$ and the projection $\bar{H} \rightarrow G_{1}$. This homomorphism must be standard since $H_{1}$ maps onto a large subgroup of $G_{1}$; for example, if $H_{1}$ is $\operatorname{Sp}(k), G_{1}$ is $\operatorname{Sp}(k+1)$ or $\operatorname{Sp}(k+2)$. Hence $G_{1} / \Gamma_{1}$ is a standard Stiefel manifold. Let $\bar{H}_{1}=\bar{G}_{1} \cap \bar{H}$. We now consider the fiber bundle $\bar{G}_{1} / \bar{H}_{1} \rightarrow{ }_{i_{1}} M \rightarrow \pi_{1} G_{1} / \Gamma_{1}$ and claim that $\pi_{1}^{*}$ is injective. Projection on the factor $G_{1}$ gives the following map of bundles:

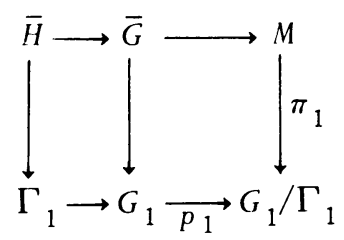

If $G_{1} / \Gamma_{1}$ is a complex or quaternionic Stiefel manifold, $p_{1}^{*}$ and hence $\pi_{1}^{*}$ is injective. In the real case $\operatorname{Spin}(q+i) / \operatorname{Spin}(q)$, if $q$ is not a power of two and $i=1$ or if $q$ and $q+1$ are not a power of two and $i=2, p_{i}^{*}$ is also injective. The other real cases when $q, q+1$ or $q+2$ is a power of two are more troublesome because of the "extra" generator $u \in H^{*}$ (Spin). Identify $u$ with an element in $H^{*}(\bar{H})$. By using the Steenrod squares of the classical groups we see that $u \notin$ Image $i^{*}$. Hence the differential map $d_{0, r}^{r+1}: H^{r}(\bar{H}) \rightarrow H^{r+1}(M)$ must take $u$ to a nonzero element for $r+1=q, q+$ 1 , or $q+2$. Suppose $q+1$ is a power of two and we are studying the case $\operatorname{Spin}(q+2) / \operatorname{Spin}(q)$. The cohomology of this Stiefel manifold is an exterior algebra on generators $v_{q}, v_{q+1}$ with $p_{1}^{*} v_{q}=w_{q}$ and $d_{0, q}^{q+1}(u)=v_{q+1}$. Since the homomorphisms must commute with the differentials, $\pi_{1}^{*} v_{q+1} \not 0$. The other cases are similar.

Even though $p_{1}^{*}$ is injective, $i_{1}^{*}$ need not be surjective. If $q_{1} \geq q$ is the first nontrivial positive dimension of $H^{*}\left(\bar{G}_{1} / \bar{H}_{1}\right)$, then from the spectral sequence we see that the first nonzero differential cannot occur until dimension $q_{1}+q-1 \geq q_{1}+11$. So $i_{1}^{*}$ is surjective through dimension $q_{1}+q-$ $2 \geq q_{1}+10$ and so $H^{*}\left(\bar{G}_{1} / \bar{H}_{1}\right)$ has no nonzero squaring operations up to that dimension. This restriction is sufficient to repeat our procedure on $\bar{G}_{1} / \bar{H}_{1}$. Let us also note that the factor $H_{1}$ is not contained in $G_{1}$. If it were, $M=G_{1} / H_{1} \times \bar{G}_{1} / \bar{H}_{1}$ which is contrary to our assumption. This means that $H_{1}$ must project nontrivially into $\bar{G}_{1}$ and so $\operatorname{Rk}\left(\bar{G}_{1}\right)-\operatorname{Rk}\left(\bar{H}_{1}\right) \geq 3$ by Lemma 2.1.

After using our procedure on $\bar{G}_{1} / \bar{H}_{1}$ we get $\bar{G}_{1}=G_{2} \times \bar{G}_{2}$ and the fiber 
bundle $\bar{G}_{2} / \bar{H}_{2} \rightarrow \bar{G}_{1} / \bar{H}_{1} \rightarrow G_{2} / \Gamma_{2}$ where the groups indexed by two have the appropriate definition and the projection induces an injection on cohomology. Now we have the bundle

$$
\bar{G}_{2} / \bar{H}_{2} \underset{i_{2}}{\longrightarrow} M \underset{\pi_{2}}{\longrightarrow} G_{1} / \Gamma_{1} \times G_{2} / \Gamma_{2}
$$

and we see that $\pi_{2}^{*}$ is injective because of the following diagram:

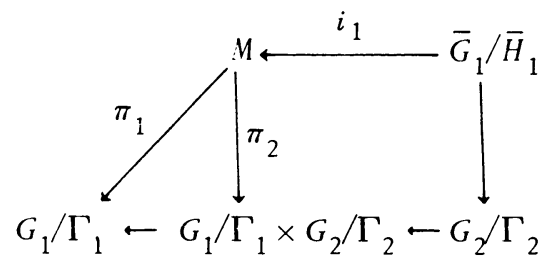

Let $q_{2} \geq q_{1}$ be the first positive, nontrivial dimension of $H^{*}\left(\bar{G}_{2} / \bar{H}_{2}\right)$. As before, $i_{2}^{*}$ is surjective through dimension $q_{2}+10$ (and so the procedure may be used again on $\left.\bar{G}_{2} / \bar{H}_{2}\right)$ and $\operatorname{Rk}\left(\bar{G}_{2}\right)-\mathrm{Rk}\left(\bar{H}_{2}\right) \geq 3$ for otherwise $M$ splits as a product.

We continue until we get the bundle

$$
\bar{G}_{s} / \bar{H}_{s} \stackrel{i_{s}}{\longrightarrow} M \stackrel{\pi_{s-1}}{\longrightarrow} G_{1} / \Gamma_{1} \times \cdots \times G_{s-1} / \Gamma_{s-1}
$$

with $\bar{G}_{s}$ and $\bar{H}_{s}$ simple, $\operatorname{Rk}\left(\bar{G}_{s}\right)-\operatorname{Rk}\left(\bar{H}_{s}\right) \geq 3$ and no Steenrod operations through dimension $q_{s}+10$. But now we reach a contradiction, for the only possibilities for $\bar{G}_{s}$ and $\bar{H}_{s}$ as listed above have rank difference less than three. Hence we must conclude that $M$ does split as a product and we get that $M$ is diffeomorphic to $\bar{G}_{1} / \bar{H}_{1} \times \cdots \times \bar{G}_{s} / \bar{H}_{s}$ where $\bar{H}_{i}=\bar{G}_{i} \cap \bar{H}$. Since $N=N_{1} \times \cdots \times N_{s}$ where $N_{i}=\bar{G}_{i} \cap N, G=\bar{G} / N=\bar{G}_{1} / N_{1} \times \cdots \times \bar{G}_{s} / N_{s}=$ $G_{1} \times \cdots \times G_{s}$ and so $M$ is diffeomorphic to $G_{1} / H_{1} \times \cdots \times G_{s} / H_{s}, H_{i}=G_{i} \cap H$. This proves Theorem 1.

Since the factors $G_{i} / H_{i}$ must have all trivial Steenrod operations and $G_{i}$ and $H_{i}$ are simple, $G_{i} / H_{i}$ can only be $\mathrm{SO}(n) / \mathrm{SO}(n-1)=S^{n-1}$, $\mathrm{SO}(n) / \mathrm{SO}(n-2)=V_{n, n-2}$ if $n$ is even, $\mathrm{SU}(n) / \mathrm{SU}(n-1)=S^{2 n-1}, \mathrm{SU}(n) / \mathrm{SU}(n-2)$ $=W_{n, n-2}$ if $n$ is even, $\mathrm{Sp}(n) / \mathrm{Sp}(n-1)=S^{4 n-1}$, or $\mathrm{Sp}(n) / \mathrm{Sp}(n-2)=X_{n, n-2}$ if $n$ is even. This establishes Theorem 2 .

If $M$ is a homotopy product of spheres, $V_{n, n-2}, W_{n, n-2}$ and $X_{n, n-2}$ cannot be a factor of $M$ by Lemma 2.3 of [7] since these spaces are not homotopy products of spheres by [3]. Hence each factor $G_{i} / H_{i}$ is a homotopy sphere. By the work of Montgomery and Samelson [4], Borel [1] and Poncet [5], each $G_{i} / H_{i}$ is a standard sphere and all the transitive actions have been classified. This means that $M$ is a product of standard spheres and if 
$G$ has an irreducibly transitive action on $M$ it is a product of the known actions on each sphere.

\section{REFERENCES}

1. A. Borel, Le plan projectif des octaves el les sphères comme espaces homogènes, C. R. Acad. Sci. Paris 230 (1950), 1378-1380. MR 11, 640.

2. - Sur l'homologie et la cohomologie des groupes de Lie compacts connexes, Amer. J. Math. 76 (1954), 273-342. MR 16, 219.

3. W. -Y. Hsiang and J. C. Su, On the classification of transitive effective actions on Steifel manifolds, Trans. Amer. Math. Soc. 130 (1968), 322-336. MR 36 \#4581.

4. D. Montgomery and H. Samelson, Transformation groups of spheres, Ann. of Math. (2) 44 (1943), 454-470. MR 5, 60.

5. J. Poncet, Groups de Lie compacts de transformations de l'espace euclidien et les sphères comme espaces homogènes, Comment. Math. Helv. 33 (1959), 109-120. MR $21 \# 2708$.

6. H. Scheerer, Homotopieäquivalente kompakte Liesche Gruppen, Topology 7 (1968), 227-232. MR 37 \# 4833.

7. Victor Schneider, Transitive actions on highly connected spaces, Proc. Amer. Math. Soc. 38 (1973), 179-185. MR 47 \#9658.

8. Emery Thomas, Exceptional Lie groups and Steenrod squares, Michigan Math. J. 11 (1964), 151-156. MR 29 \#610.

DEPARTMENT OF MATHEMATICS, UNIVERSITY OF SOUTHWESTERN LOUISIANA, LAF AYETTE, LOUISIANA 70501 